\title{
Airway Multidrug-Resistant Organisms in a Population of Tracheostomy and Chronic Ventilator-Dependent Children at a Tertiary Care Pediatric Hospital
}

\author{
Tara Havens ${ }^{1}$, David Rosen ${ }^{1}$, and Katherine Rivera-Spoljaric ${ }^{1}$ \\ ${ }^{1}$ Washington University in St Louis
}

February 11, 2022

\begin{abstract}
Background Children with tracheostomies are at an increased risk of bacterial respiratory tract infections. Infections caused by multidrug-resistant organisms (MDROs) are more difficult to treat and can result in severe complications. We investigated the risk factors and sequelae of MDRO positivity in tracheostomy and chronic ventilator-dependent children. Methods We performed a retrospective chart review of 75 tracheostomy and chronic ventilator-dependent children at St. Louis Children's Hospital. Data on demographics, respiratory cultures, hospitalizations, emergency department (ED) visits, and antibiotic usage were collected. We determined the frequency of MDRO positivity and compared the number of hospitalizations, number of ED visits, and antibiotic usage in patients with and without MDRO-positive cultures. Patient clinical variables were analyzed before and after MDRO acquisition. Results We found $75.7 \%$ (56/74) of our participants had an MDRO-positive culture, with methicillin-resistant Staphylococcus aureus (MRSA, $n=36,64 \%)$ and Pseudomonas aeruginosa $(n=8,14 \%)$ being the most commonly detected organisms. Patients with MDRO-positive cultures had a greater number of annual non-pulmonary admissions $[\mathrm{OR}=1.99,95 \% \mathrm{CI}(1.21-3.29), \mathrm{P}=0.008]$, inpatient antibiotic courses $[\mathrm{OR}=1.27,95 \% \mathrm{CI}(1.07-1.50), \mathrm{P}=0.006]$, total antibiotic courses $[\mathrm{OR}=1.26,95 \% \mathrm{CI}(1.08-1.48), \mathrm{P}=0.004]$, and chronic antibiotic use [OR=2.31, 95\% CI (1.12-4.74), $\mathrm{P}=0.03$ ] compared to MDRO-negative participants. Patients that acquired MDROs during the study period subsequently required increased outpatient antibiotics $[\mathrm{P}=0.006]$ but did not have increased pulmonary admissions or ED visits. Conclusion Frequent antibiotic usage and hospitalizations increase the risk of MDRO acquisition in children with tracheostomies and ventilator-dependence. Further antibiotic stewardship may help prevent resistant infections in technology-dependent children.
\end{abstract}

Airway Multidrug-Resistant Organisms in a Population of Tracheostomy and Chronic Ventilator-Dependent Children at a Tertiary Care Pediatric Hospital

Tara N. Havens, $\mathrm{MD}^{1}$, David A. Rosen, MD, $\mathrm{PhD}^{2,3}$, Katherine Rivera-Spoljaric, MD, MSCI ${ }^{1}$

${ }^{1}$ Division of Pediatric Allergy and Pulmonary Medicine, Washington University School of Medicine, Saint Louis, Missouri

${ }^{2}$ Division of Pediatric Infectious Diseases, Washington University School of Medicine, Saint Louis, Missouri

${ }^{3}$ Department of Molecular Microbiology, Washington University School of Medicine, Saint Louis, Missouri

Correspondence to: Tara Havens MD, Division of Allergy and Pulmonary Medicine, Department of Pediatrics, Washington University School of Medicine, St. Louis Children's Hospital, 1 Children's Pl, CB 8116, St. Louis, MO 63110., E-mail: havens.tara@wustl.edu

Keywords : pediatric, tracheostomy, multidrug-resistant organism, ventilator-dependent, antibiotics, hospitalizations 
Funding Information : No funding was provided for this study

All studies were conducted at the Department of Pediatrics, Division of Pediatric Allergy and Pulmonary Medicine, Washington University St. Louis, MO.

Running head: MDROs in tracheostomy and ventilator-dependent children

Abstract

\section{Objective/background}

Children with tracheostomies are at an increased risk of bacterial respiratory tract infections. Infections caused by multidrug-resistant organisms (MDROs) are more difficult to treat and can result in severe complications. This study aimed to investigate the risk factors and sequelae of MDRO positivity in tracheostomy and chronic ventilator-dependent children.

\section{Methods}

We performed a retrospective chart review of 75 tracheostomy and chronic ventilator-dependent children at St. Louis Children's Hospital. Data on demographics, respiratory cultures, hospitalizations, emergency department (ED) visits, and antibiotic usage were collected. We determined the frequency of MDRO positivity and compared the number of hospitalizations, number of ED visits, and antibiotic usage in patients with and without MDRO-positive cultures. Patient clinical variables were analyzed before and after MDRO acquisition.

\section{Results}

We found $75.7 \%$ (56/74) of our participants had an MDRO-positive culture, with methicillin-resistant Staphylococcus aureus (MRSA, $\mathrm{n}=36,64 \%)$ and Pseudomonas aeruginosa $(\mathrm{n}=8,14 \%)$ being the most commonly detected organisms. Patients with MDRO-positive cultures had a greater number of annual non-pulmonary admissions [OR=1.99, 95\% CI (1.21-3.29), $P=0.008$ ], inpatient antibiotic courses [OR=1.27, 95\% CI (1.07$1.50), P=0.006]$, total antibiotic courses $[\mathrm{OR}=1.26,95 \% \mathrm{CI}(1.08-1.48), P=0.004]$, and chronic antibiotic use $[\mathrm{OR}=2.31,95 \% \mathrm{CI}(1.12-4.74), P=0.03]$ compared to MDRO-negative participants. Patients that acquired MDROs during the study period subsequently required increased outpatient antibiotics $[P=0.006]$ but did not have increased pulmonary admissions or ED visits.

\section{Conclusion}

Frequent antibiotic usage and hospitalizations increase the risk of MDRO acquisition in children with tracheostomies and ventilator-dependence. Further antibiotic stewardship may help prevent resistant infections in technology-dependent children.

\section{Introduction}

Children require tracheostomies and long-term mechanical ventilation for a variety of primary pulmonary and non-primary pulmonary diagnoses. Tracheostomies bypass the respiratory tract's normal protective barriers and can serve as a conduit for bacterial colonization and subsequent infection ${ }^{1 ; 2}$. Tracheostomies are frequently colonized with bacteria which may increase the risk of respiratory tract infections including tracheitis and pneumonia ${ }^{3-5}$. Respiratory tract infections in tracheostomy and ventilator-dependent children are a frequent cause of hospitalization, morbidity, and health care utilization ${ }^{6}$. Colonization and respiratory tract infections from multidrug resistant organisms (MDROs) are a common complication in this patient population. Lower respiratory tract infections caused by MDROs can be more difficult to treat and have been associated with worse clinical outcomes including increased number of hospitalizations, increased length of hospital stay, prolonged intensive care unit stays, and overall mortality ${ }^{7-9}$.

There are no national guidelines available for treatment of respiratory tract infections in chronic ventilatordependent children $^{4 ; 6}$, and there is also paucity of medical literature on this patient population. Most 
studies in tracheostomy and ventilator-dependent children have been more descriptive in nature focusing on occurrence rates of specific organisms and demographics. Broad spectrum empiric antibiotics and chronic suppressive antibiotics are frequently used in this population and could lead to increased antibiotic resistance. Understanding which patient factors are associated with respiratory MDROs could help guide clinical decision making and antibiotic usage in this population.

\section{Materials and Methods}

We conducted a single-center retrospective study of 75 children from the tracheostomy and chronic ventilatordependent clinic at St. Louis Children's Hospital. Participants were initially enrolled from clinic for the purpose of prospectively following their clinical course with Washington University in St. Louis IRB approval, IRB \#201409115. A separate IRB submission was made for retrospective chart review for this specific study and was considered exempt, IRB \#202003084. The data obtained ranged from years 1999-2016 and were logged into a RedCap database.

\section{Data Collection}

General demographic data including primary diagnosis, gender, race, housing location, number of people in household, and ventilator use, were collected using a survey at time of enrollment as well as retrospective chart review. Primary diagnoses were put into broader categories to simplify analysis. These categories were: neuromuscular disease which included neurologic, cerebral palsy, secondary paralysis, myopathies, and muscular dystrophies; congenital anomaly: airway anomaly, congenital cardiac defect; and control of breathing: congenital central hypoventilation syndrome (CCHS), severe OSA, and rapid-onset obesity with hypothalamic dysfunction, hypoventilation, and autonomic dysregulation (ROHHAD). We performed a chart review on individual patient records to obtain data on tracheal aspirate cultures, hospitalizations, emergency department (ED) visits, time spent at transitional rehab facility, and antibiotic usage. Participants were categorized as either having a MDRO or never having MDRO on tracheal aspirate. Information on any MDRO-positive tracheal aspirates were collected including date of culture, whether it was collected during an illness, polymorphonuclear (PMN) leukocytes abundance, and species of organism. Some cultures were obtained for routine surveillance, but most tracheal aspirates were obtained at physician's discretion in response to patient symptoms.

\section{Definitions}

MDROs were defined as isolates with acquired resistance to at least one agent from three or more different classes of antimicrobials ${ }^{10}$. Antibiotic usage was categorized into number of courses of inpatient, outpatient, or chronic antibiotics (oral or inhaled) per year. Chronic antibiotics were defined as those used for $>4$ weeks and were commonly prescribed the purpose of either prophylaxis of another comorbidity (like neurogenic bladder) or suppression for tracheal colonization. Number of hospitalizations and ED visits per year were separated into pulmonary or non-pulmonary by primary reason for visit or admission. Transitional rehabilitation facility was defined by temporary inpatient admission to a local rehabilitation and subacute care hospital that facilitates transition from hospital care to home.

\section{Statistical analysis}

Descriptive and bivariate statistical analyses were used to determine MDRO incidence and to assess demographic differences between participants with MDRO-positive cultures and those without a history of cultured MDRO. To assess risk factors for MDRO positivity we used univariate mixed logistic regression to compare clinical outcome variables that occurred one year prior to MDRO acquisition versus those without MDRO. We used Wilcoxon rank sum test to make a paired comparison between pre-MDRO and post-MDRO clinical variables. We also used Wilcoxon rank sum test to assess if having MDRO positivity increases the risk of complications by comparing clinical outcomes after MDRO-positive cultures to those without a history of cultured MDRO. In secondary analysis, we excluded participants with MRSA and reanalyzed our

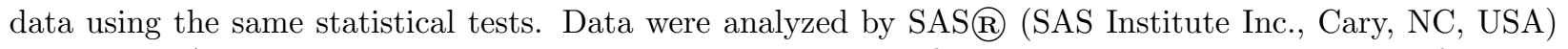
9.4 version. A $P$-value $<0.05$ was considered statistically significant. Missing data were excluded from the 
analyses.

Results

Demographics

The analysis included $74 / 75$ patients which had at least one respiratory culture in their medical record. Demographic data are presented in Table 1 including comparison between children who ever had a MDRO-positive culture and those who never had an MDRO recovered from culture throughout the study (MDRO-negative). All the participants had tracheostomies and 69 (93\%) of them were on chronic mechanical ventilation. Our patient population was predominately female and Caucasian. They mostly come from rural, small town, and suburban areas. The most common primary diagnosis was neuromuscular disease $(\mathrm{n}=45,61 \%)$ which included neurologic, cerebral palsy, secondary paralysis, myopathies, and muscular dystrophies. There was no significant difference between the baseline demographics of the MDRO-positive and MDRO-negative participants.

\section{Microbiology of Respiratory Cultures}

There was a total of $102 \mathrm{MDRO}$ positive cultures from the 74 participants. We found $75.7 \%$ (56/74) of our participants had an MDRO from a tracheal aspirate culture. Methicillin-resistantStaphylococcus aureus (MRSA, $\mathrm{n}=36,64 \%)$ and Pseudomonas aeruginosa $(\mathrm{n}=8,14 \%)$ were the most common MDROs within our population (Table 2). The median time until first MDRO after tracheostomy was 207 days and 270 days from start of chronic ventilation. Abundant ( $>25$ PMNs/LPF) PMNs ( $=24,43 \%)$ was the most common PMN Gram stain quantification identified for MDRO cultures.

\section{Risk factors for MDRO positivity}

We compared MDRO-positive patients clinical risk factors one year prior to MDRO positivity to clinical risk factors per year of those without a history of MDRO. One year prior to MDRO positivity those with MDROpositive cultures were more likely to have a greater number of non-pulmonary admissions per year $[\mathrm{OR}=1.99$, $95 \%$ CI (1.21-3.29), $P=0.008]$, more inpatient antibiotics use per year $[\mathrm{OR}=1.27,95 \%$ CI $(1.07-1.50), P$ $=0.006]$, and more IV and oral antibiotic courses both inpatient and outpatient per year $(\mathrm{OR}=1.26,95 \%$ CI (1.08-1.48), $P=0.004]$ compared to MDRO-negative participants (Table 3). MDRO-positive patients also had significantly more hospitalizations per year compared to those who are MDRO-negative [OR=2.01, 95\% CI (1.45-2.79) $P<0.001]$. MDRO-positive participants were more likely to have received chronic antibiotics (oral and inhaled) one year prior to MDRO positivity compared to those without MDRO [OR $=2.31,95 \%$ CI (1.12-4.74), $P=0.03]$ (Table 4). Though, no significant effect was found for type of chronic antibiotics, inhaled versus oral, on $\mathrm{MDRO}$ positivity [OR=1.65, 95\% CI (0.30-9.06), $P=0.67]$. Participants were divided into two groups those who were never admitted to a transitional rehab facility and those who have been admitted to transitional rehab facility. The relationship between those with and without a MDRO and previous admission to transitional rehab facility was tested and we found that admission to a transitional rehab facility was not associated with MDRO positivity [OR=2.33, 95\% CI (0.24-22.7), $P=0.36]$.

\section{Clinical Complications following MDRO positivity}

After initial MDRO positivity participants were not more likely to have increased number of hospitalizations, ED visits, or antibiotics compared to those without MDRO (Table E1). Of the MDRO-positive patients, we made a pair-wise comparison of risk factors before and after their first MDRO positivity (Table 5). Participants had more non-pulmonary admissions $(P=0.049)$ and inpatient antibiotics $(P=0.03)$ prior to having a MDRO compared to after MDRO positivity. MDRO-positive participants received more outpatient antibiotics following MDRO positivity compared to prior to MDRO positivity $(P=0.006)$.

Secondary Analysis with MRSA excluded

Since MRSA had such a high occurrence rate within our population, we reanalyzed our data without MRSA 
included. Without MRSA, Gram-negative bacilli made up the majority of the MDROs. The most common resistant organisms detected in tracheal aspirates excluding MRSA werePseudomonas aeruginosa $(\mathrm{n}=14$, $26 \%)$ followed by Achromobacter xylosoxidans $(\mathrm{n}=13,24 \%)$ and Klebsiella pneumoniae $(\mathrm{n}=9,17 \%)$ (Table E2). Overall, we had similar findings with and without MRSA included in our analysis. We again found that those who were MDRO-positive were more likely to have a greater number of non-pulmonary admissions per year $[\mathrm{OR}=2.17,95 \% \mathrm{CI}(1.11-4.22), P=0.02]$, more inpatient antibiotics use per year $[\mathrm{OR}=1.38,95 \%$ CI (1.13-1.69), $P=0.002]$, and more IV and oral antibiotic courses per year compared to MDRO-negative participants [OR=1.31, 95\% CI (1.09-1.57), $P=0.004]$ (Table E3). In contrast to the analysis with MRSA included, in our analysis without MRSA we found that participants who are MDRO-positive also had more pulmonary admissions per year $[\mathrm{OR}=2.07,95 \%$ CI $(1.17-3.67), P=0.01]$. Using a pair-wise comparison participants had more non-pulmonary admissions $(P=0.04)$ and inpatient antibiotics $(P=0.02)$ prior to having a MDRO compared to after MDRO positivity (Table E4). Unlike in our full analysis we did not find that participants receive more outpatient antibiotics following MDRO positivity $(P=0.20)$.

\section{Discussion}

Tracheostomy and ventilator-dependent children are frequently prescribed broad spectrum empiric antibiotics for illnesses $7 ; 11 ; 12$. Frequent and prolonged use of antibiotics is associated with emergence of antibiotic resistance in numerous patient populations ${ }^{13-15}$, but there is a paucity of data specific to tracheostomy and ventilator-dependent children. Our study found that increased antibiotic usage and episodes of hospitalization put these patients at risk of MDROs. Other studies have found respiratory tract MDROs increase the risk of complications ${ }^{1 ; 8} ; 9 ; 16$, but we did not find that our participants had more complications following MDRO detection.

We had a higher rate of MDROs in our patient population compared to other studies of tracheostomy and ventilation-dependent children ${ }^{15}$. $76 \%$ of our participants had at least one respiratory tract culture with a MDRO. Our participants were predominantly dependent on mechanical ventilation (93\%) which could explain the higher rate of MDROs in our group ${ }^{15 ; 17 ; 18}$. MRSA was the most common MDRO within our patient population with $49 \%$ of participants positive. This was consistent with a recent publication by McCaleb et al. that found a similar rate of MRSA within their single center study of pediatric tracheostomy patients ${ }^{19}$. S. aureus and Pseudomonas aeruginosa are the most commonly cultured organisms from tracheostomies of pediatric patients $8 ; 9 ; 18 ; 19$. Russell et al. found that tracheostomies in pediatric patients increase risk of Gram-negative organisms such as P. aeruginosa but not $S$. aureus ${ }^{8}$. It is suspected that organisms found in the normal flora of the respiratory tract, like S. aureus, are protective against lower respiratory tract infections but organisms such as Pseudomonas disrupt the normal flora which can lead to more infections ${ }^{20}$. Gram-negative organisms are clearly associated with an increased risk of lower respiratory tract infections, but it is less clear if MRSA cultured from the airway represents colonization versus evidence of a lower respiratory tract infection.

We found that frequent hospitalizations and antibiotic usage increased the risk of MDRO in the respiratory tract of technology-dependent children. It has been shown that hospitalizations and prior antibiotic usage were independent risk factors for lower respiratory tract MDROs in adults, but has not been well described in children ${ }^{15}$. We also found that both intermittent antibiotic usage and chronic antibiotics increased risk of MDRO. We did not find that there was an independent effect from type of chronic antibiotic used, inhaled or oral, on risk of MDRO positivity. The literature is unclear about emergence of antibiotic resistance with inhaled antibiotics. Long term use of inhaled antibiotics in cystic fibrosis patients increases the mean inhibitory concentration (MIC) over time but does not affect the clinical efficacy of antibiotic treatment ${ }^{21-23}$. Furthermore, inhaled antibiotics in cystic fibrosis have not been found to select for intrinsically resistant organisms ${ }^{21-23}$. This differs from a review by Laska et al. which demonstrated that use of inhaled antibiotics increased the risk of resistant organisms in patients with bronchiectasis ${ }^{24}$. There remains little literature describing emergence of antibiotic resistance with inhaled antibiotics in the pediatric tracheostomy and ventilator-dependent population. 
Our study did not find having an MDRO increased the risk of complications including number of hospitalizations, ED visits, or antibiotic usage compared to those without MDRO. This finding contrasts several papers that found having resistant Gram-negative bacilli orPseudomonas to be a risk factor for increased length of stay, increased number of lower respiratory tract infections, and mortality ${ }^{1 ; 8 ; 9 ; 16}$. In our analysis without MRSA, Gram-negative bacilli were the most common type of resistant organism cultured. We did not find an increased risk of complications following MDRO positivity with the exclusion of MRSA from our analysis. We found that participants received more outpatient antibiotics after MDRO positivity compared to prior to MDRO positivity using a pair-wise comparison of these groups. This increased use of antibiotics could be evidence of more frequent respiratory tract infections following MDRO detection. It has been shown that specific organisms such as P. aeruginosa, Enterobacteriaceae, or Acinetobacter baumannii in tracheal cultures are associated with an increased number of lower respiratory tract infections $7 ; 8$. The increased use of antibiotics seen following MDRO detection in our group may also represent physicians having a lower threshold to treat with antibiotics when an MDRO is found. Even when an MDRO is cultured from the respiratory tract it is hard to determine if the organism represents colonization or a true infection. One of the challenges in treating tracheostomy-dependent children is defining true infection and treating appropriately.

There are several limitations to this study. Our study is a single center retrospective chart review, so the data are less generalizable. This study is also limited by a relatively small sample size which may lead to underpowered statistical analyses. There is considerable variability in care provided including when tracheal aspirate cultures were obtained, when antibiotics were used, and thresholds for hospital admission. Data were limited only to what could be accessed in our electronic health medical record. We aimed to maximize the data collected for each patient by manually extracting data instead of using automated data collection software.

Despite these limitations our study offers insight into a less-studied pediatric technology-dependent patient population. Tracheostomy and ventilator-dependent children represent a group of patients with complex medical needs and high health care utilization. Respiratory tract infections including tracheitis and pneumonia are the most common cause of hospitalization within this population ${ }^{3-5}$. There are no guidelines published for treatment and management of respiratory tract infections in this patient population. Broad spectrum empiric antibiotics are frequently used to treat respiratory tract infections in these children but could increase risk of MDRO acquisition. Our study suggests that more antibiotic usage and hospitalizations may increase risk of MDRO positivity. This specific group of patients could benefit from increased antibiotic stewardship focus with a tailored antibiotic strategy. Additional studies are needed to help develop guidelines for treatment of respiratory tract infections in tracheostomy and ventilator-dependent children with the goal of minimizing the emergence of MDROs.

Acknowledgments : We thank Hongjie Gu, MS and Michael Wallendorf, PhD who provided statistical analysis through Division of Biostatistics at Washington University School of Medicine.

\section{References}

1. Niederman MS, Ferranti RD, Zeigler A, Merrill WW, Reynolds HY. 1984. Respiratory infection complicating long -term tracheostomy. The implication of persistent gram-negative tracheobronchial colonization. Chest . 85(1):39-44.

2. Toews GB, Hansen EJ, Strieter RM. 1990. Pulmonary host defenses and oropharyngeal pathogens. The American Journal of Medicine . 88(5, Supplement 1):S20-S24.

3. Yu H, Mamey MR, Russell CJ. 2017. Factors associated with 30-day all-cause hospital readmission after tracheotomy in pediatric patients.Int J Pediatr Otorhinolaryngol. 103:137-141.

4. Zhu H, Das P, Roberson DW, Jang J, Skinner ML, Paine M, Yuan J, Berry J. 2015. Hospitalizations in children with preexisting tracheostomy: A national perspective. The Laryngoscope . 125(2):462-468.

5. Russell CJ, Mamey MR, Koh JY, Schrager SM, Neely MN, Wu S. 2018. Length of stay and hospital revisit after bacterial tracheostomy-associated respiratory tract infection hospitalizations. Hosp Pediatr. 8(2):72-80. 
6. Russell CJ, Mack WJ, Schrager SM, Wu S. 2017. Care variations and outcomes for children hospitalized with bacterial tracheostomy-associated respiratory infections. Hospital Pediatrics . 7(1):16-23.

7. Grosse-Onnebrink J, Rudloff J, Kessler C, Werner C, Dougherty GW, Kerschke L, Kahl BC, Omran H. 2019. Acinetobacter baumannii is a risk factor for lower respiratory tract infections in children and adolescents with a tracheostomy. Pediatr Infect Dis J . 38(10):1005-1009.

8. Russell CJ, Simon TD, Mamey MR, Newth CJL, Neely MN. 2017.Pseudomonas aeruginosa and posttracheotomy bacterial respiratory tract infection readmissions. Pediatr Pulmonol . 52(9):1212-1218.

9. Sanders CD, Guimbellot JS, Muhlebach MS, Lin F-C, Gilligan P, Esther CR. 2018. Tracheostomy in children: Epidemiology and clinical outcomes.Pediatric Pulmonology . 53(9):1269-1275.

10. Magiorakos A-P, Srinivasan A, Carey RB, Carmeli Y, Falagas ME, Giske CG, Harbarth S, Hindler JF, Kahlmeter G, Olsson-Liljequist B et al. 2012. Multidrug-resistant, extensively drug-resistant and pandrugresistant bacteria: An international expert proposal for interim standard definitions for acquired resistance. Clinical Microbiology and Infection . 18(3):268-281.

11. Rusakow LS, Guarin M, Wegner CB, Rice TB, Mischler EH. 1998. Suspected respiratory tract infection in the tracheostomized child: The pediatric pulmonologist's approach. Chest. 113(6):1549-1554.

12. Goldman JL, Newland JG, Price M, Yu D, Lee BR. 2019. Clinical impact of an antimicrobial stewardship program on high-risk pediatric patients.Infect Control Hosp Epidemiol . 40(9):968-973.

13. CDC. 2019. Centers for Disease Control and Prevention: Antibiotic resistance threats in the United States, 2019. https://www.cdc.gov/drugresistance/pdf/threats-report/2019-ar-threats-report-508.pdf.

14. Murray MT, Beauchemin MP, Neu N, Larson EL. 2019. Prior antibiotic use and acquisition of multidrug-resistant organisms in hospitalized children: A systematic review. Infect Control Hosp Epidemiol . 40(10):1107-1115.

15. Chen G, Xu K, Sun F, Sun Y, Kong Z, Fang B. 2020. Risk factors of multidrug-resistant bacteria in lower respiratory tract infections: A systematic review and meta-analysis. Can J Infect Dis Med Microbiol. 2020:7268519.

16. Nathwani D, Raman G, Sulham K, Gavaghan M, Menon V. 2014. Clinical and economic consequences of hospital-acquired resistant and multidrug-resistant Pseudomonas aeruginosa infections: A systematic review and meta-analysis. Antimicrob Resist Infect Control . 3(1):32.

17. Kuster SP, Hasse B, Huebner V, Bansal V, Zbinden R, Ruef C, Ledergerber B, Weber R. 2010. Risks factors for infections with extended-spectrum beta-lactamase-producing escherichia coli and Klebsiella pneumoniae at a tertiary care university hospital in switzerland. Infection . 38(1):33-40.

18. McLaren D, Chitakis M, Burns H, Kapur N. 2021. Airway microbiology in tracheostomized children. Respiratory Care. 66(2):281-285.

19. McCaleb R, Warren RH, Willis D, Maples HD, Bai S, O'Brien CE. 2016. Description of respiratory microbiology of children with long-term tracheostomies. Respir Care. 61(4):447-452.

20. Harlid R, Andersson G, Frostell CG, Jorbeck HJ, Ortqvist AB. 1996. Respiratory tract colonization and infection in patients with chronic tracheostomy. A one-year study in patients living at home.American journal of respiratory and critical care medicine.154(1):124-129.

21. Smith S, Rowbotham NJ, Regan KH. 2018. Inhaled anti-pseudomonal antibiotics for long-term therapy in cystic fibrosis. Cochrane Database of Systematic Reviews . (3).

22. Oermann CM, Retsch-Bogart GZ, Quittner AL, Gibson RL, McCoy KS, Montgomery AB, Cooper PJ. 2010. An 18-month study of the safety and efficacy of repeated courses of inhaled aztreonam lysine in cystic fibrosis. Pediatr Pulmonol . 45(11):1121-1134. 
23. Burns JL, Van Dalfsen JM, Shawar RM, Otto KL, Garber RL, Quan JM, Montgomery AB, Albers GM, Ramsey BW, Smith AL. 1999. Effect of chronic intermittent administration of inhaled tobramycin on respiratory microbial flora in patients with cystic fibrosis. J Infect Dis.179(5):1190-1196.

24. Laska IF, Crichton ML, Shoemark A, Chalmers JD. 2019. The efficacy and safety of inhaled antibiotics for the treatment of bronchiectasis in adults: A systematic review and meta-analysis. The Lancet Respiratory Medicine. 7(10):855-869.

\section{Hosted file}

Tables and Figures MDROs in tracheostomy and ventilator-dependent children .docx available at https://authorea.com/users/460083/articles/556152-airway-multidrug-resistantorganisms-in-a-population-of-tracheostomy-and-chronic-ventilator-dependent-children-ata-tertiary-care-pediatric-hospital 